\title{
Appendiceal carcinoma associated with microsatellite instability
}

\author{
ANGÉLICA MORALES-MIRANDA ${ }^{1}$, ISMAEL DOMÍNGUEZ ROSADO ${ }^{2}$, \\ CARLOS CHAN NÚÑEZ ${ }^{2}$ and FREDY CHABLE MONTERO ${ }^{3}$
}

\begin{abstract}
Departments of ${ }^{1}$ Reproductive Biology and ${ }^{2}$ Surgery, National Institute of Medical Sciences and Nutrition Salvador Zubirán; ${ }^{3}$ Department of Pathology, Hospital Médica Sur, Mexico City 14080, Mexico
\end{abstract}

Received November 14, 2017; Accepted March 2, 2018

DOI: $10.3892 / \mathrm{mco} .2018 .1596$

\begin{abstract}
Approximately 15\% of colorectal cancer (CRC) cases exhibit microsatellite instability (MSI), which appears to be associated with unique biological behavior. The present study presents a case of appendiceal carcinoma associated with MSI that responded well to adjuvant chemotherapy. Clinical, pathological and immunohistochemical (IHC) characteristics have been described. The 60 -year-old male patient had suffered from recurrent lower abdominal pain associated with abdominal distention for 6 months; then, following an acute attack, he was subjected to laparoscopic appendectomy. The histopathological examination revealed moderately differentiated appendiceal adenocarcinoma with mucinous areas, without lymphovascular or perineural invasion. The IHC examination was positive for keratin-20 and caudal type homeobox 2, and negative for MutL Homolog 1, MutS Homolog (MSH) 2 and MSH-6. A postoperative colonoscopy revealed diverticulosis, without the presence of polyps or tumors. However, an abdominal axial computerized tomography scan revealed thickening of the distal portion of the appendix, increased density of the greater omentum, and metastases to the liver capsule, spleen and peritoneum. The treatment of choice was right hemicolectomy with peritoneal debulking, followed by 10 cycles of chemotherapy with 5-fluorouracil, leucovorin and oxaliplatin (FOLFOX regimen). After 5 years of follow-up, the patient remains in good condition, without clinical or radiological signs of recurrence. The good response to chemotherapy corresponds with the observations made in other colon cancers with MSI. Therefore, testing for MSI in appendiceal carcinomas may provide useful information on prognosis and predict response to chemotherapy.
\end{abstract}

Correspondence to: Dr Angélica Morales-Miranda, Department of Reproductive Biology, National Institute of Medical Sciences and Nutrition Salvador Zubirán, Avenue Vasco de Quiroga 15 Col, Belisario Domínguez, Section XVI, Tlalpan, Mexico City 14080, Mexico

E-mail: angelica.moralesm@incmnsz.mx; angelica170969@aol.com

Key words: appendiceal adenocarcinoma, microsatellite instability, prognosis, peritoneal metastases

\section{Introduction}

Microsatellite instability (MSI) is a type of genetic instability resulting from alterations in the DNA mismatch repair (MMR) system. The microsatellites are mono-, di- or trinucleotides, distributed in non-coding regions of the human genome. Approximately $15 \%$ of all colorectal adenocarcinomas (CRCs) display MSI, which occurs due to a germline mutation in one of the MMR genes, such as $h M S H 2$ (2p16), hMSH6 (2p16), hPMS1 (2q31), hPMS2 (7p22) or TGF $\beta R I I$, or to epigenetic silencing of $h M L H 1$ (3p21). Of these mutations, the most common are those in the genes encoding the hMSH2 and hMLH1 proteins, whereas hypermethylation of $M L H 1$ occurs in only 2-3\% of CRCs (1-5). CRC is the second cause of digestive tract cancer-related mortality. According to the International Classification of Disease (ICD-10, revised in 2009), primary epithelial neoplasms of the appendix are grouped within CRC. This type of cancer develops at the tip or base of the appendix, and has an incidence of $0.082 \%$. The peak incidence of epithelial neoplasms is usually between 50 and 60 years of age, and their association with sex is controversial. The symptoms of appendiceal carcinoma tend to be subtle, particularly in the early stages (6-10). Appendiceal tumors include adenomas, adenocarcinomas, mucinous neoplasms, undifferentiated carcinoma, small-cell carcinoma, and signet ring cell carcinoma (SRCC). Some classify cystadenocarcinomas and non-cystic tumors separately $(11,12)$. Mucinous adenocarcinomas represent $\sim 45 \%$ of all appendiceal tumors $(13,14)$. The peritoneal spread of mucin allows such deposits to accumulate in particular areas, including the greater omentum, the undersurface of the right hemidiaphragm, the pelvic cavity and the right retrohepatic space. This redistribution is the result of tumor cell accumulation at the sites where ascitic fluid is reabsorbed in the abdomen. The selection of the surgical procedure depends on the type of appendiceal carcinoma, as well as the location and size of the tumor mass. The recommended treatment is right hemicolectomy followed by combined adjuvant intraperitoneal chemotherapy, supplemented by additional cycles of systemic chemotherapy (15-18). The reported 5-year survival for appendiceal carcinoma patients ranges between 20 and 60\%, depending on the degree of invasion of parenchymal organs and/or regional lymph node metastases (19-21). CRC with MSI has distinctive characteristics, including the tendency to arise in the proximal colon, as well as poor differentiation, extensive lymphocytic infiltration, presence of signet ring cells and abundant mucin. 
Patients with MSI tumors appear to have a better prognosis compared with those with microsatellite stable tumors (22); however, the response to 5-fluorouracil (5-FU)-based chemotherapy regimens are poorer with MSI tumors $(23,24)$. At present, little information is available regarding the prevalence of MSI in appendiceal adenocarcinoma.

\section{Case report}

A 62-year-old man consulted a private physician due to a 6-month history of diffuse abdominal pain (4/10 intensity) of the colic type, radiating toward the right iliac fossa, right testicle and thigh. The pain was associated with distension and accompanied by chills; there was no reported nausea, vomiting, fever, or changes in the evacuation pattern. Palpation of the abdominal region did not reveal any masses. One week after the clinical symptoms worsened, including pain intensification, an axial computerized tomography (CT) scan of the abdominal area was performed, revealing increased density of the greater omentum. An inflammatory process was ruled out at this stage. The results of routine laboratory analyses, including hematological and hepatic function tests, were normal. The measurement of carcinoembryonic antigen detected a level of $<2.0 \mathrm{mcg} / \mathrm{l}$ (normal, $0-2.5 \mathrm{mcg} / \mathrm{l}$ ) during the diagnosis, treatment and follow-up of the patient. Exploratory laparoscopy and appendectomy were performed, and the pathological examination of the surgical specimen revealed an uncommon appendicular lesion, namely tubulovillous adenocarcinoma.

The postoperative period was uneventful, with complete resolution of the abdominal pain. The patient was discharged from the hospital in a good condition. One week later, the patient was subjected to colonoscopy, which revealed no traces of suspicious lesions. Two months later, the patient was admitted to the Memorial Sloan-Kettering Cancer Center (MSKCC; New York, NY, USA) for a new evaluation and continuation of treatment. Based on the evaluation, it was recommended that the patient underwent debulking surgery. During the surgery, new lesions were identified in the peritoneum, as well as under the spleen, leading to a splenectomy and removal of the tail of the pancreas. In the immediate postoperative period, an abdominal CT scan revealed a pancreatic fistula, which was treated conventionally. Since the new investigations revealed a bifurcated pancreas, it was not possible to administer intraperitoneal chemotherapy. Nine months later, an adjuvant chemotherapy scheme was initiated, with FOLFOX-6 for 10 cycles.

Laboratory and imaging studies were performed initially every 4 months during the first 2 years, and every 6-8 months thereafter. After 5 years of follow-up, the patient remains asymptomatic with no signs of tumor activity, and attends follow-up visits every 12 months at the MSKCC.

\section{Radiology report}

Thorax. No signs of mediastinal adenopathy or pulmonary infiltration were observed. In the inferior lobe of the left lung, a $12.8-\mathrm{mm}$ nodule of non-specific origin with well-defined borders was identified.

First CT scan of the abdomen and pelvis. Areas of high density were observed in the peritoneal fat, suggestive of secondary metastases. The liver parenchyma exhibited low-density round lesions, $3.5 \mathrm{~mm}$ in diameter, with cystic characteristics, located in segments VII and VI. Multiple diverticula were identified in the left and sigmoid colon.

Second CT scan of the abdomen and pelvis. An increase in density of the greater omentum was detected, along with free liquid adjacent to the descending colon and thickening of the distal portion of the cecal appendix.

\section{Diagnostic methodology}

Laparotomy. An appendectomy was performed, revealing an inflammatory appendix with a retrocecal location and turbid purulent liquid of inflammatory origin, along with omentitis.

Pathological examination. The appendix measured $6.1 \times 0.9 \mathrm{~cm}$ and had a smooth external surface. Upon sectioning, A $2.5-\mathrm{cm}$ mass was encountered in the distal third of the organ. The mass contained solid light-brown and microcystic components, the latter being filled with mucin (Fig. 1A and B).

Microscopic findings. The mucinous neoplasm was composed of benign tubular glands and papillary structures (90\%), the latter projecting into the lumen. The glands and papillae were lined by a tall columnar epithelium, which exhibited nuclear pseudostratification with mild pleomorphism (Fig. 1C).

Part of the neoplasm (10\%) consisted of small, medium-sized, dilated and infiltrating glands that invaded the appendicular wall and reached the subserosa (Fig. 1D). The invasive component was lying in a sclerotic stroma and was accompanied by hyperplastic lymphoid tissue (Fig. 1E).

The peritoneum displayed extracellular mucin deposits, consistent with localized peritoneal adenomucinosis (Fig. 1F). The diagnosis was moderately differentiated adenocarcinoma with mucinous areas, arising in a mucinous tubulopapillary adenoma. Selected sections were obtained and embedded in paraffin for IHC analysis. The following antibodies were used: Cytokeratin (CK) 20, CDX2, MLH-1, MSH-2 and MSH-6.

The intestinal phenotype of tubulopapillary adenoma and invasive adenocarcinoma was confirmed by positivity for CK20 and CDX2 (Fig. 1G). The adenocarcinoma was negative for MLH-1, MSH-2 and MSH-6 (Fig. 1H). The pattern correlates with a mismatch repair-deficient tumor or MSI-H.

Diagnosis. The following diagnosis was made for the patient: i) Moderately differentiated adenocarcinoma (10\% of the tumor) with mucinous areas, infiltrating up to the subserosa (pT3), originating in a tubulovillous adenoma (90\% of the tumor) of the distal third of the cecal appendix, with a largest diameter of $2.5 \mathrm{~cm}$. ii) Peritoneal adenomucinosis located in the periappendicular region (1 focus). iii) Surgical border (appendicular and mesoappendix) free of tumor. iv) No lymphovascular or perineural invasion.

\section{Materials and methods}

IHC analysis. IHC staining was applied to identify CDX-2, MLH-1, MSH-2 and MSH-6 expression. To prevent the non-specific binding of antibodies, the sections were blocked 

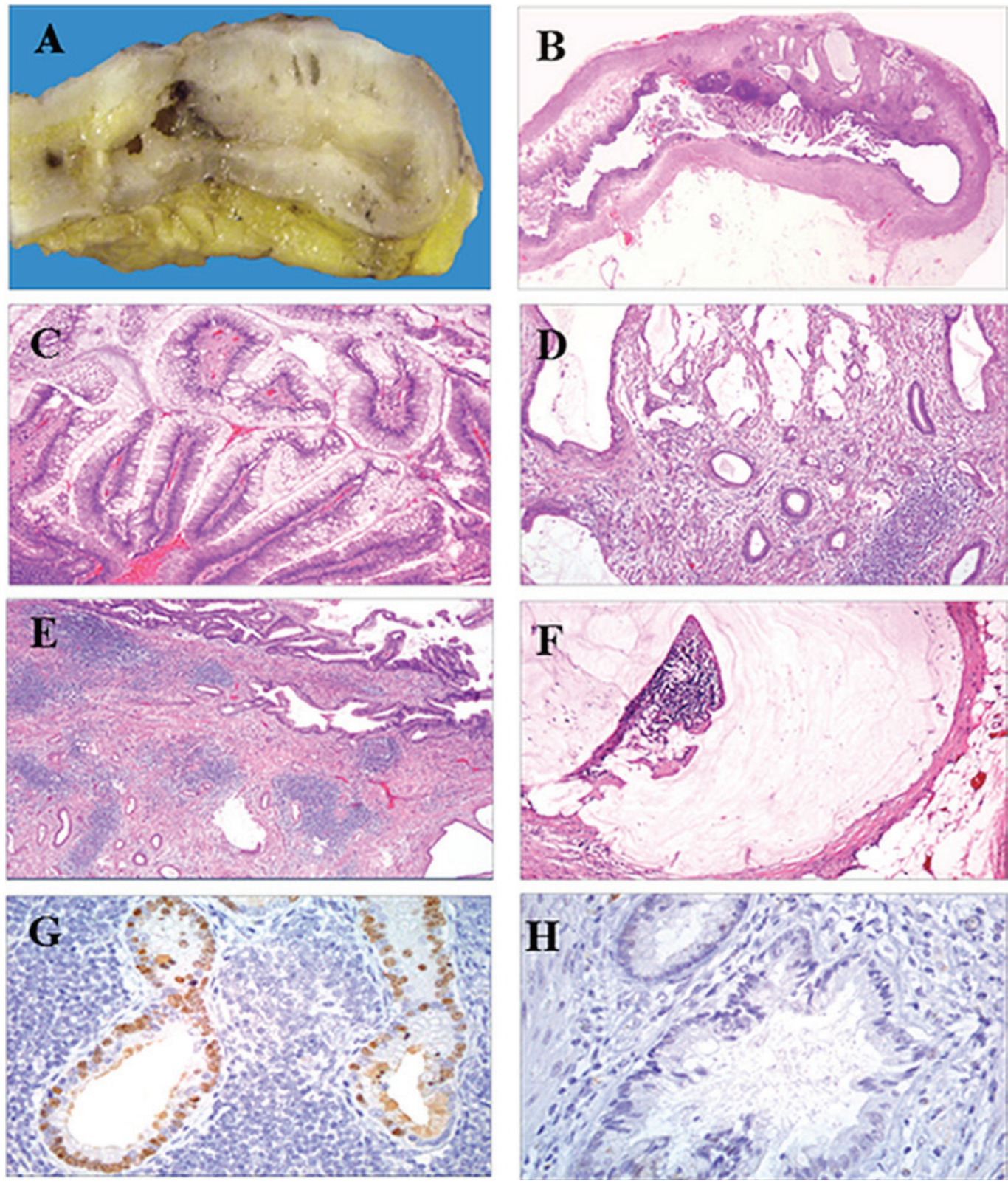

Figure 1. Cross section of the appendix, exhibiting solid light-brown and microcystic areas filled with mucin (panel A). Whole mounted section of the appendix displaying a papillary intraluminal benign neoplasm and an invasive mucin-secreting adenocarcinoma infiltrating into the subserosa (panel B). Papillary component of the mucinous adenoma, exhibiting papillae lined by tall columnar epithelial cells with nuclear pseudostratification and mild pleomorphism (panels C and D). The mucinous invasive adenocarcinoma was composed of small and medium-sized dilated malignant glands lined by columnar epithelium with mild-to-moderate pleomorphism, and was accompanied by a sclerotic stroma and hyperplastic lymphoid tissue (panel E). Localized peritoneal adenomucinosis, with the serosa displaying a focus of extracellular mucin deposit, without an epithelial component (panel F). The invasive intestinal-type adenocarcinoma was positive for CDX2 (panel G). The invasive adenocarcinoma was negative for MLH-1, MSH-2 and MSH-6 (panel H). Magnification, x4 (panel B), x10 (panel C-F) and x20 (panel G-H).

with bovine serum albumin (BSA) (Sigma-Aldrich Co., St. Louis, MO, USA) for $60 \mathrm{~min}$. The slides were then incubated with the primary antibody overnight at $4^{\circ} \mathrm{C}$ for the detection of anti-CDX-2 (AMT-28, sc-56818; dilution 1:25), anti-MLH-1 (N-20, sc-581; dilution 1:25), anti-MSH-2 (H-300, sc-22771; dilution 1:50) (Santa Cruz Biotechnology, Santa Cruz, CA, USA), anti-MSH-6 (HPA028446; dilution 1:100), and anti-CK-20 (SAB4502249; dilution 1:300) (Sigma-Aldrich), followed by incubation with the appropriate secondary antibody (Mouse/Rabbit-ImmunoDetector-HRP Cat. BSB0003-BioSB), at 1:200 dilution for $60 \mathrm{~min}$ at $37^{\circ} \mathrm{C}$. The sections were washed and stained with 3,3'-diaminobenzidine tetrahydrochloride (DAB) chromogen (Zymed ${ }^{\circledast / I n v i t r o g e n ~ I n c ., ~ C a r l s b a d, ~ C A, ~}$
USA), and hematoxylin was used for nuclear counterstaining (MHS-1, Sigma-Aldrich). The sections were mounted with coverslips and synthetic mounting medium (Entellan; Merck, Darmstadt, Germany; OB046327).

In each case, negative controls were included that lacked the primary antibody. Images were captured on a Nikon-Eclipse 80i microscope coupled to a Nikon digital sight camera (Melville, New York, NY, USA).

\section{Discussion}

Adenocarcinoma of the appendix is rare, constituting $<1 \%$ of all CRCs. Appendiceal tumors may exhibit atypical clinical 
characteristics, representing a challenge for diagnosis and treatment. Appendiceal neoplasms are seldom detected before or during appendectomy, with $<1.5 \%$ of the appendectomy specimens harboring primary appendiceal cancers upon examination (25). CRC is divided into two general groups, having either genomic pathways or MSI pathways.

The first group comprises $75-80 \%$ of all CRCs, while the tumors with MSI constitute $15-20 \%$ of all cases. MSI, reflecting inactivation of the MMR genes, is present in nearly all cancers from individuals with hereditary non-polyposis colorectal cancer (HNPCC). The prevalence of MSI-H in appendiceal carcinomas, reported at $2.8 \%$, is associated with germline mutations.

MSI is usually associated with poorly differentiated, mucin-producing tumors that are generated by epithelial cells, giving rise to peritoneal carcinomatosis. MSI linked to colorectal tumors is divided into two different phenotypes, denominated as high (MSI-H) and low (MSI-L). The tumors with MSI-H are more susceptible to treatment by chemotherapy, and are thus associated with better survival. However, MSI-L tumors are more resistant to adjuvant chemotherapy. Currently, the use of adjuvant chemotherapy is extrapolated from the beneficial effect of this treatment on colorectal cancer, although the validity of this extrapolation is uncertain. The largest cohort of appendiceal adenocarcinoma patients to date, which was recently reported, demonstrated that there was a relative protective effect for patients who received systemic chemotherapy compared with those who did not, applicable to both the mucinous and non-mucinous histological types (26). Due to the low ERCC1 expression observed in appendiceal adenocarcinomas, platinum agents, such as cisplatin or oxaliplatin, may be combined with either 5 -FU or gemcitabine. Data in the literature indicate that the most effective regimen following surgical resection of advanced (stage II and III) CRC is FOLFOX, comprising 5-FU, leucovorin and oxaliplatin. This triad reduces the risk of recurrence and improves survival. In the present report of a case of appendiceal carcinoma, the patient received FOLFOX for 6 months after recovering from a pancreatic fistula.

Over the last few years, new developments include intraoperative hyperthermic intraperitoneal chemotherapy (27) with mitomycin at $40^{\circ} \mathrm{C}$, and early postoperative intraperitoneal chemotherapy with 5-FU. These are considered to be effective adjuvant treatments for minimal residual disease after debulking. The long-term survival rate of patients undergoing complete debulking and having a low-grade tumor is $80 \%$, whereas this rate is $\sim 45 \%$ at 20 years in patients with high-grade tumors. In a small cohort of 40 patients with appendiceal mucinous neoplasms (AMNs) diagnosed between 2000 and 2011, where tissue microarrays were constructed from a representative block of the primary tumor, CDX2 single-staining revealed a sensitivity of $93 \%$ and a specificity of $56 \%$ for AMNs, whereas CK20 single-staining demonstrated a sensitivity of $98 \%$ and a specificity of $50 \%$ for AMNs (28). CDX2 is both a sensitive and specific marker of intestinal differentiation, and it is overexpressed in CRC cells compared with normal intestinal epithelium; it is a potential marker of the effectiveness of surgical resection or other local treatment modalities $(29,30)$.
Cytoreductive surgery is a new technique employing peritonectomy procedures to eradicate peritoneal carcinomatosis; judging by the absence of lymph node and other metastases, this treatment favorably affects the prognosis of survival for CRC patients.

Systematic reviews of CRC prognosis have demonstrated that tumors exhibiting a MSI phenotype have a better prognosis compared with those with a microsatellite stable pattern. The improved prognosis in the hypermutated cases may be associated with a strong immune response to the tumor caused by the expression of neoantigens that cause an in situ lymphocytic reaction (31). In the present case, MSI was determined by the absence of specific nuclear staining against three mismatch repair proteins (MLH1, MSH2 and MSH6). Tumors displaying this pattern are MSI-H, or mismatch repair-deficient. The predictive value of the MSI status to chemotherapy is important due to the variable response, particularly in cases with reported resistance to 5-FU (32). In this report, the patient received 10 cycles of the standard adjuvant treatment (FOLFOX) after surgical resection, with a good response during the 5-year post-treatment clinical follow-up. Although more prospective studies are required to correlate the prognosis with response to adjuvant therapy in rare colon cancers, such as appendiceal carcinoma, we consider that the molecular characterization of the tumor, particularly the microsatellite pattern, may help with the selection of the therapeutic scheme.

\section{Acknowledgements}

The authors would like to thank Dr Dulce Maria Carrillo for the review of the literature.

\section{Funding}

No funding was received.

\section{Availability of data and materials}

The findings are contained within the manuscript. Originals are kept in the medical records.

\section{Authors' contributions}

AMM was responsible for the study design, the preparation of the manuscript and the draft the final manuscript. FCM performed the pathology studies and prepared the figure for the article. IDR and $\mathrm{CCN}$, both surgeon physicians, contributed to the discussion and reviewed the manuscript.

\section{Ethics approval and consent to participate}

Not applicable.

\section{Consent for publication}

Written informed consent was obtained from the patient.

\section{Competing interests}

The authors declare that they have no competing interests. 


\section{References}

1. Samowitz WS, Curtin K, Ma KN, Schaffer D, Coleman LW, Leppert M and Slattery ML: Microsatellite instability in sporadic colon cancer is associated with an improved prognosis at the population level. Cancer Epidemiol Biomarkers Prev 10: 917-923, 2001

2. Stadler ZK: Diagnosis and management of DNA mismatch repair-deficient colorectal cancer. Hematol Oncol Clin North Am 29: 29-41, 2015.

3. Jacob S and Praz F: DNA mismatch repair defects: Role in colorectal carcinogenesis. Biochimie 84: 27-47, 2002.

4. Syngal S, Fox EA, Eng C, Kolodner RD and Garber JE: Sensitivity and specificity of clinical criteria for hereditary non-polyposis colorectal cancer associated mutations in MSH2 and MLH1 J Med Genet 37: 641-645, 2000.

5. Taggart MW, Galbincea J, Mansfield PF, Fournier KF, Royal RE, Overman MJ, Rashid A and Abraham SC: High-level microsatellite instability in appendiceal carcinomas. Am J Surg Pathol 37 : 1192-1200, 2013.

6. Andersson A, Bergdahl L and Boquist L: Primary carcinoma of the appendix. Ann Surg 183: 53-57, 1976.

7. Sugarbaker PH: Epithelial appendiceal neoplasms. Cancer J 15 : 225-235, 2009

8. Hananel N, Powsner E and Wolloch Y: Adenocarcinoma of the appendix: An unusual disease. Eur J Surg 164: 859-862, 1998.

9. Iwuagwu OC, Jameel JK, Drew PJ, Hartley JE and Monson JR: Primary carcinoma of the appendix-Hull series. Dig Surg 22: $163-167,2005$

10. Cerame MA: A 25-year review of adenocarcinoma of the appendix. A frequently perforating carcinoma. Dis Colon Rectum 31: 145-150, 1988.

11. Turaga KK, Pappas SG and Gamblin T: Importance of histologic subtype in the staging of appendiceal tumors. Ann Surg Oncol 19: $1379-1385,2012$

12. O'Donnell ME, Badger SA, Beattie GC, Carson J and Garstin WI: Malignant neoplasms of the appendix. Int J Colorectal Dis 22: 1239-1248, 2007.

13. Misdraji J and Young RH: Primary epithelial neoplasms and other epithelial lesions of the appendix (excluding carcinoid tumors). Semin Diagn Pathol 21: 120-133, 2004.

14. Feizi I, Maleki N and Tavosi Z: Mucinous adenocarcinoma of the appendix. J Res Med Sci 20: 103-104, 2015.

15. Chua TC, Pelz JO and Morris DL: Surgery for colorectal peritoneal carcinomatosis. Scand J Gastroenterol 47: 277-285, 2012.

16. Varisco B, McAlvin B, Dias J and Franga D: Adenocarcinoid of the appendix: Is right hemicolectomy necessary? A meta-analysis of retrospective chart reviews. Am Surg 70: 593-599, 2004

17. Proulx GM, Willett CG, Daley W and Shellito PC: Appendiceal carcinoma: Patterns of failure following surgery and implications for adjuvant therapy. J Surg Oncol 66: 51-53, 1997.

18. McConnell YJ, Mack LA, Gui X, Carr NJ, Sideris L, Temple WJ, Dubé P, Chandrakumaran K, Moran BJ and Cecil TD: Cytoreductive surgery with hyperthermic intraperitoneal chemotherapy: An emerging treatment option for advanced goblet cell tumors of the appendix. Ann Surg Oncol 21: 1975-1982, 2014

19. Ung L, Chua TC and David LM: Peritoneal metastases of lower gastrointestinal tract origin: A comparative study of patient outcomes following cytoreduction and intraperitoneal chemotherapy. J Cancer Res Clin Oncol 139: 1899-1908, 2013.
20. Nash GM, Smith JD, Tang L, Weiser MR, Temple LK, O'Reilly E, Saltz LB, Guillem JG and Paty PB: Lymph node metastasis predicts disease recurrence in a single-center experience of 70 stages 1-3 appendix cancers: A retrospective review. Ann Surg Oncol 22: 3613-3617, 2015.

21. Samdani T, Schultheis M, Stadler Z, Shia J, Fancher T, Misholy J, Weiser MR and Nash GM: Lymph node yield after colectomy for cancer: Is absence of mismatch repair a factor? Dis Colon Rectum 58: 288-293, 2015.

22. Kelly KJ: Management of appendix cancer. Clin Colon Rectal Surg 28: 247-255, 2015.

23. Ribic CM, Sargent DJ, Moore MJ, Thibodeau SN, French AJ, Goldberg RM, Hamilton SR, Laurent-Puig P, Gryfe R. Shepherd LE, et al: Tumor microsatellite-instability status as a predictor of benefit from fluorouracil-based adjuvant chemotherapy for colon cancer. N Engl J Med 349: 247-257, 2003.

24. Carethers JM, Smith EJ, Behling CA, Nguyen L, Tajima A, Doctolero RT, Cabrera BL, Goel A, Arnold CA, Miyai K and Boland CR: Use of 5-fluorouracil and survival in patients with microsatellite-unstable colorectal cancer. Gastroenterology 126: 394-401, 2004

25. Xie X, Zhou Z, Song Y, Li W, Diao D, Dang C and Zhang H: The management and prognostic prediction of adenocarcinoma of appendix. Sci Rep 6: 39027, 2016.

26. Asare EA, Compton CC,HannaNN,Kosinski LA, Washington MK, Kakar S, Weiser MR and Overman MJ: The impact of stage, grade, and mucinous histology on the efficacy of systemic chemotherapy in adenocarcinomas of the appendix: Analysis of the National Cancer Data Base. Cancer 122: 213-221, 2016.

27. Glockzin G, Gerken M, Lang SA, Klinkhammer-Schalke M, Piso P and Schlitt HJ: Oxaliplatin-based versus irinotecan-based hyperthermic intraperitoneal chemotherapy (HIPEC) in patients with peritoneal metastasis from appendiceal and colorectal cancer: A retrospective analysis. BMC Cancer 14: 807, 2014.

28. Alsaad KO, Serra S, Schmitt A, Perren A and Chetty R: Cytokeratins 7 and 20 immunoexpression profile in goblet cell and classical carcinoids of appendix. Endocr Pathol 18: 16-22, 2007.

29. Wong SC, Ng SS, Cheung MT, Luk LY, Chan CM, Cheung AH, Lee VH, Lai PB, Ma BB, Hui EP, et al: Clinical significance of CDX2-positive circulating tumour cells in colorectal cancer patients. Br J Cancer 104: 1000-1006, 2011.

30. Liu Q, Teh M, Ito K, Shah N, Ito Y and Yeoh KG: CDX2 expression is progressively decreased in human gastric intestinal metaplasia, dysplasia and cancer. Mod Pathol 20: 1286-1297, 2007.

31. Smyrk TC, Watson P, Kaul K and Lynch HT: Tumor-infiltrating lymphocytes are a marker for microsatellite instability in colorectal carcinoma. Cancer 91: 2417-2422, 2001

32. Arnold CN, Goel A and Boland CR: Role of hMLH1 promoter hypermethylation in drug resistance to 5-fluorouracil in colorectal cancer cell lines. Int J Cancer 106: 66-73, 2003. 